\title{
Test of a theory of predictive behavior in young children
}

RICHARD S. BOGARTZ

INSTITUTE OF CHILD BEHAVIOR AND DEVELOPMENT, UNIVERSITY OF IOWA

Data are presented in support of a theory of predictive behavior in young children which emphasizes the role of attention and response-and event-produced stimulus traces, and deemphasizes the role of learning in the prediction of binary event sequences by young children.

In the binary prediction situation, $S$ 's task is to predict which of two events will occur on each of a series of trials by making one of two predictive responses (PR) each time a cue occurs. When preschool children predict the next event in a single alternation sequence they almost all make errors. They do not always pay attention to the events they have just predicted, in that during the interval in which the event occurs they may be looking away and not see it. Such inattending often coincides with an error run in which the events alternate ABAB ... while the child is predicting BABA ... on the same trials. Apparently at times the child simply alternates his previous PR regardless of the event. Termination of such a run of errors is often preceded by a reorientation to the event during the final error trial, accompanied by a verbal or nonverbal response which suggests $S$ has noticed he made an error. Errors also occur on trials in which the child takes a long time to predict, appears indecisive, and considers aloud the two alternatives before responding, suggesting that at times the $P R^{\prime}$ 's are guesses.

\section{Theory}

Assume that each $P R$ produces a stimulus trace, $t_{r}$, conditioned to the alternate $P R$, and that if $t_{r}$ is still present when the cue for the next PR occurs, it elicits that alternate PR. Assume that if $S$ makes an attending response ( $A R$ ) to the event, the $t_{r}$ is displaced by a trace of the event, $t_{e}$, and that if $t_{e}$ is still present when the next cue occurs, it elicits the PR to which it is conditioned, prediction of the alternate event. Also assume that $t_{r}$ and $t_{e}$ are vulnerable to distracting stimuli which displace them, and that if no trace is present when the cue occurs, $S$ guesses at random. (The conditioning states of the traces, 1 . e., the rules determining which response a given trace will elicit, are taken to be-determined by preexperimental associations, associations established during pretraining sessions, or learning which is completed during the early trials of the experiment.) In everyday language, the assumptions say that on each trial $\mathbf{S}$ guesses at random unless he remembers his previous $P R$ or the event just presented. If he remembers the PR, he makes the alternate PR; if he remembers the event, he predicts the alternate event. To remember the event he must have paid attention, and if he remembers the event he does not remember his response.

If now it is also assumed that on each trial the probability of an AR is $p$, the probability of displacement of $t_{r}$ by distracting stimuli is $d_{1}$, and the probability of displacement of $t_{e}$ by distracting stimuli if the AR does occur is $d_{2}$, then the stochastic process and its associated probability measure shown in Fig. 1 describes the trial-to-trial response transitions. Recalling that the sequence of events is a single alternation sequence, it immediately follows that the sequence of correct responses and errors will be a twostate first order Markov chain with stationary transition probabilities. The entries in the transition matrix of this chain will be $\mathrm{p}_{\mathrm{cc}}=1-1 / 2 \gamma, \mathrm{p}_{\mathrm{ce}}=1 / 2 \gamma, \mathrm{p}_{\mathrm{ec}}=a+1 / 2 \gamma$, and $\mathrm{p}_{\mathrm{ee}}=\beta+1 / 2 \gamma$, where $\mathrm{p}_{\mathrm{i}}$ is the probability of a transition from a response of type $i$ on trial $\mathrm{n}$ to a response of type $\mathrm{j}$ on trial $\mathrm{n+1}$, and where $a=\mathrm{p}\left(1-\mathrm{d}_{2}\right)$, $\beta=(1-p)\left(1-d_{1}\right)$, and $\gamma=1-a-\beta$. Obtaining maximum likelihood estimates of $\alpha$ and $\beta$, and therefore of $\gamma$ (Anderson \& Goodman, 1957), and also an estimate of the initial probability of a correct response, it is possible to predict the values of various sequential statistics both for the group data and for individual Ss (Bogartz, in press).

\section{Method}

Twenty 4- and 5-year-old children from the University Preschool Laboratories predicted on 100 consecutive trials the color of the next marble in an alternating sequence of two colors randomly selected from the colors Black, Blue, White, and Yellow. Each S was seated before a marble-dispensing apparatus and told that he was going to play a marble game. He was instructed to guess the color of the next marble each time the buzzer sounded and to press the marble-release switch only after making his guess. He was shown a pair of marbles having the colors which would be presented and asked to identify their colors. Then $\mathrm{S}$ was told that that pair of colors would be used in the game but that first he would practice with red marbles. Five practice trials were given in which $S$ predicted the red marble each time the buzzer sounded and then obtained a red marble by pressing the switch. The $\mathbf{S}$ was again shown the two colors and reminded that only they would occur. The 100 trials were then run without interruption, with the buzzer sounding for $.3 \mathrm{sec}$. every $8 \mathrm{sec}$.

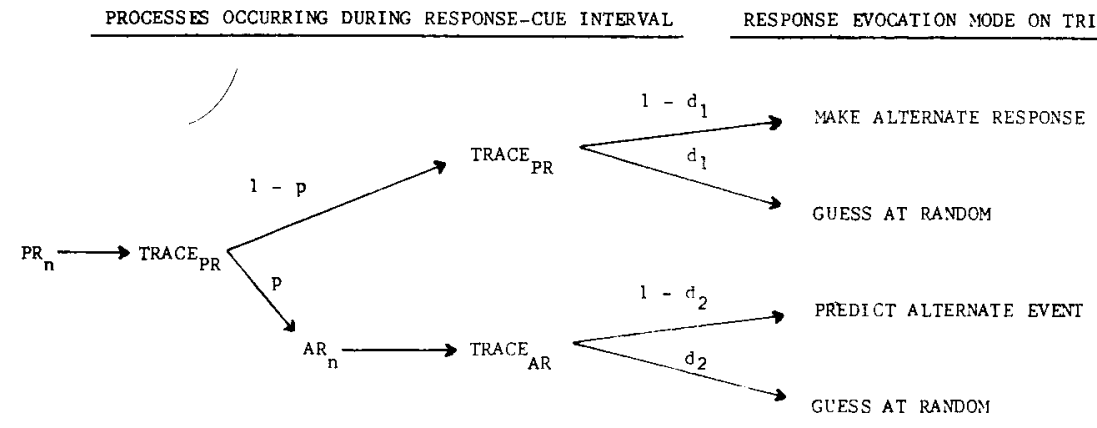

Fig. 1. The stochastic process and probability measure characterizing the trial-to-trial response transitions. 
Table 1. Comparison of observed relative frequencies of 3-tuples with relative frequencies predicted using the same parameter values for all Ss

\begin{tabular}{lllllllll} 
& ccc & cce & cec & cee & ecc & ece & eec & eee \\
\hline Pred. & .536 & .113 & .091 & .045 & .119 & .025 & .047 & .023 \\
Obs. & .560 & .096 & .095 & .042 & .097 & .041 & .042 & .027 \\
\hline
\end{tabular}

\section{Results}

A $x^{2}$-test of homogeneity of parameter values within the group of 20 Ss gave a $\chi^{2}$ of 171.93 on $38 \mathrm{df}$, equivalent to a $z$ of 9.88 . The individual sequences, therefore, almost certainly are not samples from the same first order Markov chain, consequently group $x^{2}$-tests of Order and Stationarity are of little value since they presuppose samples from a single chain. There is interest, nevertheless, in the extent to which the model implied by the theory described above predicts the group data even when there is heterogeneity of parameter values. Table 1 shows the observed relative frequencies with which the eight possible 3-tuples occurred throughout the 20100 trial protocols. The predicted values were obtained using a single parameter set for the entire group. Apparently the group data are predicted well even when the parameter values vary among the individual Ss.

Table 2 and Fig. 2 show that the model is predicting well the behavior of the individual Ss. Table 2 shows that averaging over the individual predicted values obtained by estimating a parameter set for each $S$ results in smaller deviations of predicted values from the observed group means for the 10 sequential statistics than does predicting on the basis of a single parameter set. This is true also for the total number of runs of errors for which the observed mean value was 13.95 , Pred ${ }^{G}$ was 14.44, and Pred. ${ }_{A}$ was 13.74.

Figure 2 shows the scatterplot of observed vs. predicted total number of error runs for the $20 \mathrm{Ss}$, indicating that the result in Table 2 is not due to cancellation of large but opposite discrepancies for the individual Ss. Inspection of the observed and predicted values of $r_{j}$ and $\mathrm{C}_{\mathrm{k}}$ for the individual Ss revealed goodness-of-fit comparable to that shown in Fig. 2.

\section{Discussion}

The results provide substantial support in general for

Table 2. Observed and predicted mean values of $r_{j}$, the number of error runs of length $j$, and $c_{k}$, the number of joint occurrences of two correct responses $k$ trials apart, with Pred. $G$ obtained by using one set of parameters for the entire group, and Pred. ${ }_{A}$ obtained by averaging over individual $\mathrm{Ss}$ the predicted values obtained with individual parameter estimates.

\begin{tabular}{rrrrrrrr} 
& Pred. G & Pred.A & Obs. & & Pred.G & Pred.A & Obs. \\
\hline$r_{1}$ & 9.72 & 9.39 & 9.80 & $C_{1}$ & 64.27 & 65.02 & 64.80 \\
$r_{2}$ & 3.18 & 2.73 & 2.25 & $C_{2}$ & 61.52 & 63.23 & 64.20 \\
$r_{3}$ & 1.04 & .95 & 1.30 & $C_{3}$ & 60.56 & 62.34 & 61.50 \\
$r_{4}$ & .34 & .37 & .45 & $C_{4}$ & 59.88 & 61.63 & 62.15 \\
$r_{5}$ & .11 & .16 & .15 & $C_{5}$ & 59.24 & 60.96 & 60.20 \\
\hline
\end{tabular}

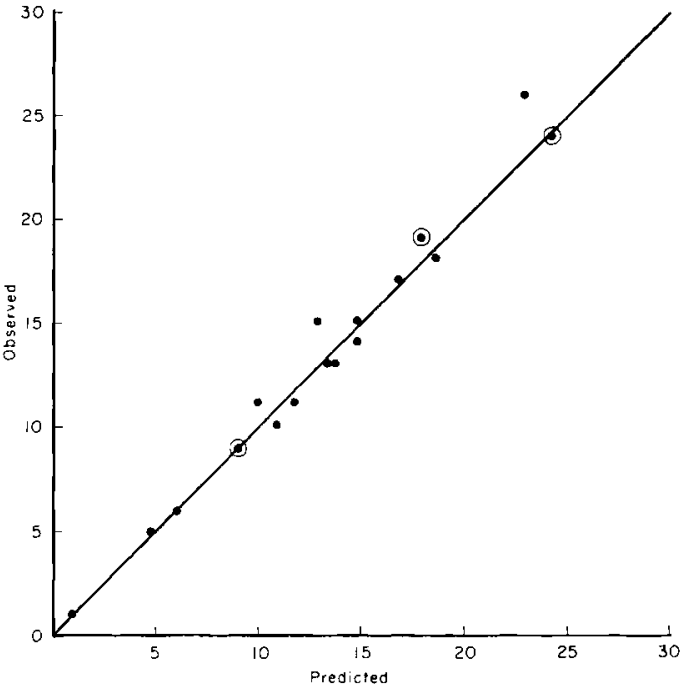

Fig. 2. Scatterplot of the observed $v s$. predicted total number of error runs for the $20 \mathrm{Ss}$.

the class of theories which imply a two-state first order Markov chain with stationary transition probabilities as a model of the sequence of correct responses and errors, and in particular for the theory described above. The latter, which views the child as a system that generates its output by tracking either the PR or the event on the previous trial or by switching to a random output generator when off track, provides an alternative to learning theories which thus far have swept the field with respect to theoretical formulations of performance changes in the binary prediction situation. The present theory predicts a mean performance curve which is of the same exponential form as that arising in a number of learning models (e.g., Estes, 1959), although it focuses not on trial-to-trial changes in the conditioning states of the stimulus or stimuli, but rather on trial-to-trial changes in the source and type of stimulus which elicits the PR. This theory does provide a role for early, rapid conditioning of the stimulus traces, and of course would require their reconditioning to explain performance changes which would follow transfer to a new type of sequence. The questions which will be at issue if the present theory is shown to have generality will be when and how fast does learning take place, and must it be assumed to be occurring throughout the entire session in the binary prediction situation, or will other mechanisms be more appropriate to the explanation of most of the trial-to-trial changes in behavior.

\section{References}

Anderson, T. W., \& Goodman, L. A. Statistical inference about Markov chains. Ann. Math. Stat., 1957, 28, 89-1101

Bogartz, R. S. Theorems for a finite sequence from a two-state first order Markov chain with stationary transition probabilities. Psychometrika, 1966, in press.

Estes, W. K. Component and pattern models with Markovian interpretations. In R. R. Bush \& W. K. Estes (Eds.), Studies in mathematical learning theory. Stanford: Stanford University Press, 1959. Pp. 9-52. 